\title{
Bistable auto-aggregation phenotype in Lactiplantibacillus plantarum emerges after cultivation in in vitro colonic microbiota
}

\author{
Julia Isenring ${ }^{1}$, Annelies Geirnaert ${ }^{1}$, Christophe Lacroix ${ }^{1 *}$ and Marc J. A. Stevens ${ }^{1,2}$
}

\begin{abstract}
Background: Auto-aggregation is a desired property for probiotic strains because it is suggested to promote colonization of the human intestine, to prevent pathogen infections and to modulate the colonic mucosa. We recently reported the generation of adapted mutants of Lactiplantibacillus plantarum NZ3400, a derivative of the model strain WCFS1, for colonization under adult colonic conditions of PolyFermS continuous intestinal fermentation models. Here we describe and characterize the emerge of an auto-aggregating phenotype in L. plantarum NZ3400 derivatives recovered from the modelled gut microbiota.
\end{abstract}

Results: L. plantarum isolates were recovered from reactor effluent of four different adult microbiota and from spontaneously formed reactor biofilms. Auto-aggregation was observed in L. plantarum recovered from all microbiota and at higher percentage when recovered from biofilm than from effluent. Further, auto-aggregation percentage increased over time of cultivation in the microbiota. Starvation of the gut microbiota by interrupting the inflow of nutritive medium enhanced auto-aggregation, suggesting a link to nutrient availability. Auto-aggregation was lost under standard cultivation conditions for lactobacilli in MRS medium. However, it was reestablished during growth on sucrose and maltose and in a medium that simulates the abiotic gut environment. Remarkably, none of these conditions resulted in an auto-aggregation phenotype in the wild type strain NZ3400 nor other non-aggregating L. plantarum, indicating that auto-aggregation depends on the strain history. Whole genome sequencing analysis did not reveal any mutation responsible for the auto-aggregation phenotype. Transcriptome analysis showed highly significant upregulation of LP_RS05225 ( $\mathrm{msa}$ ) at 4.1-4.4 $\log _{2}$-fold-change and LP_RS05230 (marR) at 4.5-5.4 $\log _{2}$-fold-change in all auto-aggregating strains compared to non-aggregating. These co-expressed genes encode a mannose-specific adhesin protein and transcriptional regulator, respectively. Mapping of the RNA-sequence reads to the promoter region of the msa-marR operon reveled a DNA inversion in this region that is predominant in autoaggregating but not in non-aggregating strains. This strongly suggests a role of this inversion in the auto-aggregation phenotype.

Conclusions: L. plantarum NZ3400 adapts to the in vitro colonic environment by developing an auto-aggregation phenotype. Similar aggregation phenotypes may promote gut colonization and efficacy of other probiotics and should be further investigated by using validated continuous models of gut fermentation such as PolyFerms.

\footnotetext{
*Correspondence: christophe.lacroix@hest.ethz.ch

1 Laboratory of Food Biotechnology, Institute of Food, Nutrition

and Health, ETH Zurich, Schmelzbergstrasse 7, 8092 Zürich, Switzerland

Full list of author information is available at the end of the article
} 
Keywords: Auto-aggregation, Lactiplantibacillus plantarum, In vitro gut microbiota, Adaptation, Transcriptome, DNA inversion

\section{Background}

Consumption of probiotics increased steadily over the past years and their application was recommended against a range of gastrointestinal tract related diseases [1]. Probiotics are defined as "live microorganisms that, when administered in adequate amounts, confer a health benefit on the host "[2]. To exert a health benefit, a probiotic should exhibit certain properties such as survival during gastrointestinal passage, delivery of high viable-cell numbers to the colon and a certain degree of colonization in the gut environment $[3,4]$. Successful colonization in the intestine is mediated via adherence to the intestinal epithelium or solid particles.

Auto-aggregation occurs between genetically identical cells and co-aggregation between genetically different cells [5]. Auto-aggregation is a well-studied phenomenon that leads to the formation of a community structure that facilitates interaction and communication between cells, genetic exchange, adherence and colonization in different environments [6-11]. Auto-aggregation ability correlates positively with adherence to human epithelial cell lines and the ability to co-aggregate with Listeria monocytogenes, Staphylococcus aureus and pathogenic Escherichia coli $[8,12-16]$. Auto-aggregation is frequently assessed as feature in studies evaluating putative probiotic strains [17-19].

Although auto-aggregation is a common phenomenon, there is still no complete understanding of underlying mechanisms and triggers, possibly impeded by species-specific differences. Triggering factors of autoaggregation so far identified include intestinal, nutritive, chemical, and oxidative stresses, changes in temperature and nutrient availability [20-25]. Several autoaggregation mechanisms have been reported, including cell-surface properties, -structures and -enzymes. Cell surface hydrophobicity for example is positively linked to auto-aggregation [14, 26, 27]. Molecules involved in auto-aggregation, so called autoagglutinins, include cell-surface proteins, exopolysaccharides, carbohydrates, glycoproteins, teichoic and lipoteichoic acid secreted proteins that act as aggregation promoting factors $[7,23,28,29]$.

Auto-aggregation was reported for Lactobacillus spp. strains isolated from distinct environments like the piglet, chicken and murine gastrointestinal tract, vaginal tract, dairy and fermented foods [12, 30-37]. However, not much is known about bacterial auto-aggregation and its role in the human intestinal tract. Lactiplantibacillus plantarum WCFS1 is a model strain for probiotic lactobacilli. It harbors possible genetic predisposition for auto-aggregation in form of a serine/threonine rich domain in LP_RS01260 with affinity to mucin which was identified to be involved in auto-aggregation of L. plantarum NCIMB 8826, the mother strain of WCFS1 [38, 39]. However, there is no description of a WCFS1 autoaggregation phenotype yet. Recently, we supplemented $L$. plantarum NZ3400, a derivative strain of WCFS1, to different in vitro human colonic microbiota, continuously cultivated in the PolyFermS model in the context of an evolutionary engineering experiment [40]. L. plantarum NZ3400 derivatives were recovered from the microbiota and phenotypically and genotypically characterized. Here, we describe the emerge of an auto-aggregation phenotype in a non-aggregating strain after exposure to human colon conditions. The novel phenotype was characterized, and transcriptome analysis was performed to investigate the underlying mechanisms.

\section{Results}

\section{Development of a high-throughput screening} for auto-aggregation

A high-throughput screening method based on a yeastagglutination assay was set-up to detect auto-aggregating L. plantarum strains [41]. Microscopic investigation of 96L. plantarum cultures grown in a 96-well tissue culture test plate revealed that auto-aggregating L. plantarum cultures produced a sediment of white clumps, while non-aggregating cultures formed a homogenous white layer at the bottom of the wells (Fig. 1A). Therefore, detection of clumps can be used to distinguish rapidly between auto-aggregating and non-aggregating strains.

To determine how many L. plantarum isolates have to be analyzed to obtain a reliable percentage of the autoaggregation subpopulation, different sets of colonies isolated from a single reactor and time point were visually assigned as aggregating or not. Testing 48, 96, 144 and 192 colonies did not significantly change aggregation percentage (Fig. 1B). Therefore, 48 colonies were investigated in all further experiments to determine auto-aggregation percentages.

\section{L. plantarum auto-aggregation increases in biofilm, during starvation and during cultivation in in vitro colonic microbiota}

To investigate if auto-aggregation is microbiota-dependent, L. plantarum NZ3400 was supplemented to four 


\section{A

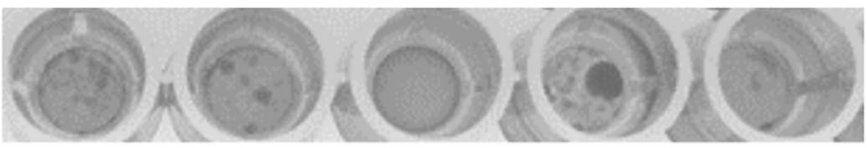 \\ $\begin{array}{lllll}1 & 2 & 3 & 4 & 5\end{array}$ \\ B Determination of auto-aggregation \\ for different sample sizes}

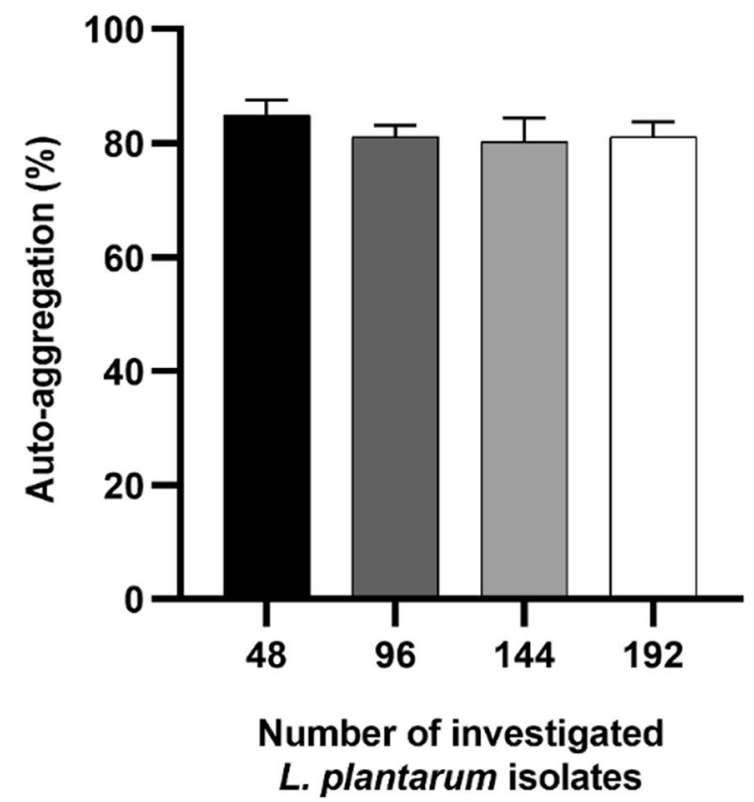

Fig. 1 Visual auto-aggregation detection and determination of the number of colonies to investigate auto-aggregation. A 96-well plate well bottom appearance of non-aggregating (well 3) and auto-aggregating (well 1-2,4-5) L. plantarum isolates. B Percentage of auto-aggregation after testing different numbers of L. plantarum colonies

PolyFermS models inoculated with different colonic microbiota and recovered after 10 days. Auto-aggregation was detected in L. plantarum isolates recovered from all four microbiota at levels between 8 and $41 \%$ (Fig. 2A). Auto-aggregation percentage steadily increased over time in five different reactors containing microbiota of donor 2 , reaching approximately 15 and $30 \%$ after 21 and 42 days post supplementation, respectively (Fig. 2B). An increase in auto-aggregation over time was also observed for donor 3 microbiota. It was more pronounced compared to donor 2 microbiota in the reactors 1,3 and 4 with $88-89 \%$ aggregation 18 days post $L$. plantarum supplementation, but lower in the reactors 2 and 5 with only $8 \%$ aggregation after 18 days (Fig. 2 C).

Further, biofilms spontaneously formed on the reactor walls were collected at the end of fermentations.
The auto-aggregation percentage of $L$. plantarum strains from biofilms was at least two times higher than from reactor effluent at the final day of fermentation (Fig. 2B), suggesting a role of auto-aggregation in biofilm establishment. However, no correlation was found between auto-aggregation percentage and L. plantarum colonization level in the reactor (data not shown). Since aggregation might facilitate the formation of biofilms, biofilm formation ability of the wild type and the aggregating, isogenic strain PA4_02 on polystyrene was assessed using crystal violet and optical density measurement. Strains were grown compared in MRS and minimal medium containing different carbon sources. The aggregating strain PA4_02 showed clearly increased biofilm formation compared to the wild type when grown in minimal medium supplemented 


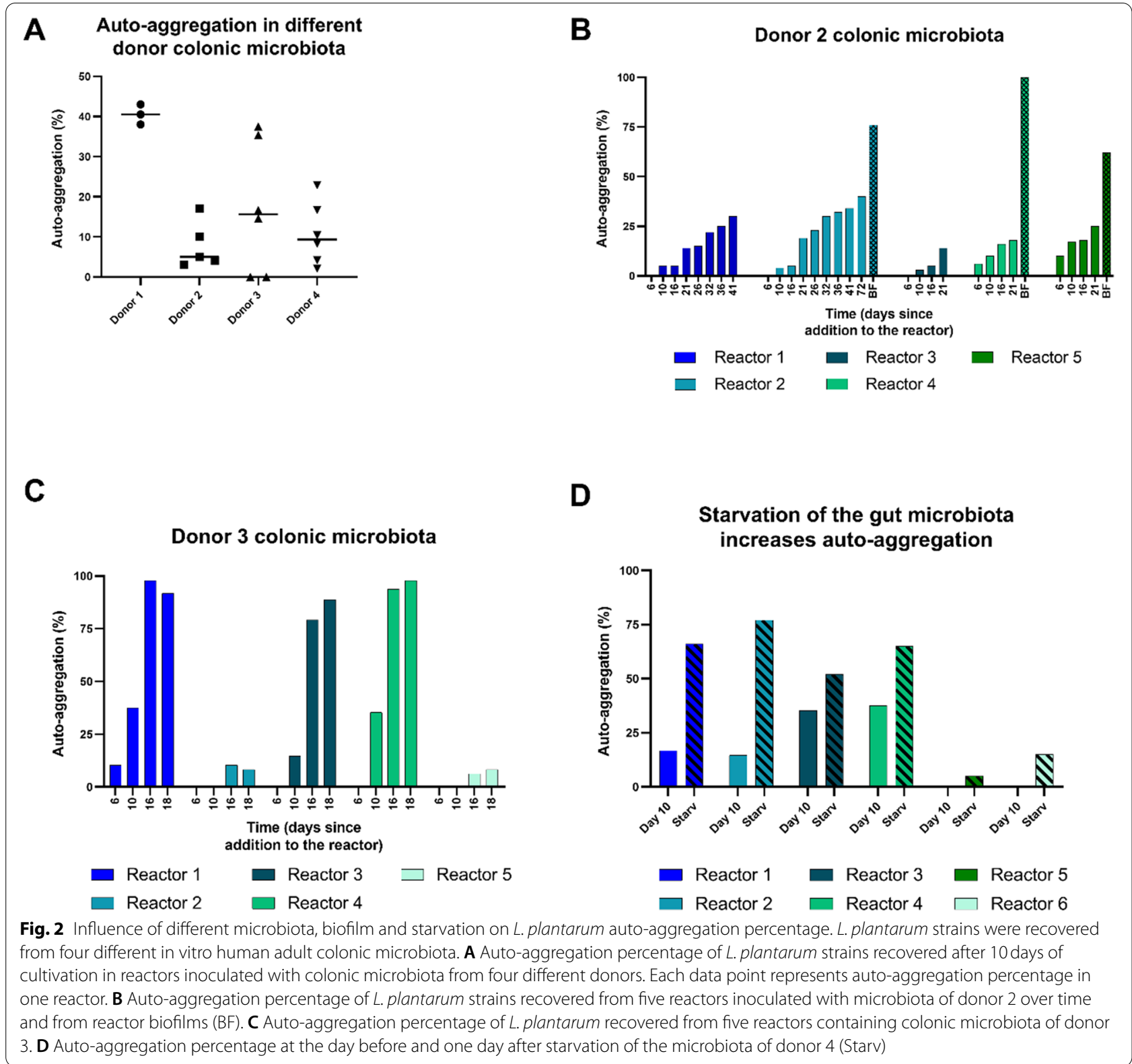

with maltose but not in any other conditions (Additional file 1: Figure S1).

Auto-aggregation is suggested to be linked to cellproximity during nutrient exchange or to nutritive stress [23]. Therefore, starvation of colonic microbiota was simulated by interrupting the medium inflow for $24 \mathrm{~h}$ in six TRs containing microbiota of donor 4. Starvation led to accumulation of isobutyrate, isovalerate and valerate, which indicates enhanced protein fermentation (Additional file 2: Figure S2). Auto-aggregation percentage increased during starvation in all reactors (Fig. 2D). Moreover, after starvation, auto-aggregation was even detected in reactors where no auto-aggregation was observed before the starvation period (Fig. 2D, reactor 5 and 6). Simultaneously, L. plantarum viable cell counts decreased by 1-2 logs during starvation (data not shown).

\section{Bistability of auto-aggregation phenotype depends on nutrient availability}

To investigate the stability of the auto-aggregation phenotype, fifteen $L$. plantarum NZ3400 derivatives with auto-aggregation phenotype, recovered from all four microbiota, were serially cultured in MRS medium daily 
for 3 days. All strains still exhibited a strong auto-aggregation phenotype that was clearly visible after the first overnight culture (Fig. 3A). Auto-aggregation was not visually detectable anymore after the second overnight culture, yet small aggregates were still observed microscopically (Fig. 3B). Finally, aggregates were only barely detectable microscopically in the third culture (Fig. 3C). Conclusively, auto-aggregation is instable under nutrientrich cultivation conditions, hinting again towards a role of nutrient availability in auto-aggregation.

Thereafter, L. plantarum strains that lost their aggregation capacity upon consecutive cultivation in MRS medium were grown in a minimal medium supplemented with different carbon sources and in EMS, a medium that simulates the abiotic environment of the reactor gut microbiota [40]. L. plantarum strains IA01, PA4_02 and PA1.2_01 lost their auto-aggregation capacity in repeated MRS cultures but recovered this capacity when regrown in EMS medium and in minimal medium containing sucrose or maltose, but not in the same medium with glucose as sole carbon source. Remarkably, L. plantarum NZ3400 and four isolates (Table 1) recovered from the modelled microbiota of donor 1 and 2 that never showed auto-aggregation, did not auto-aggregate in any of the tested conditions. These results show that different nutrient conditions as such do not result in an autoaggregation phenotype in L. plantarum NZ3400 and its derivatives, but that auto-aggregation is only observed in strains that have been exposed to the gut microbiota.

\section{Genome and transcriptome analyses of auto-aggregating L. plantarum isolates}

The possibility to reestablish auto-aggregation only in isolates that were exposed to modelled colonic microbiota suggests a lasting genomic modification responsible for the phenotype. However, whole genome sequencing analysis did not reveal any shared single nucleotide polymorphisms nor genetic reorganization in seven auto-aggregating isolates compared to NZ3400. Moreover, three thereof did not harbor any mutation and were thus isogenic to L. plantarum NZ3400 (data not shown). Because genome sequences did not provide an explanation for the auto-aggregation phenotype, transcriptome analysis using RNA sequencing was performed to identify genes possibly involved in the auto-aggregation phenotype.

The transcriptome of the wild type strain NZ3400 was compared to that of the strain $I A 01_{\mathrm{t} 0}$ during growth in MRS, conditions under which AI01 aggregates and NZ3400 does not. The comparison revealed

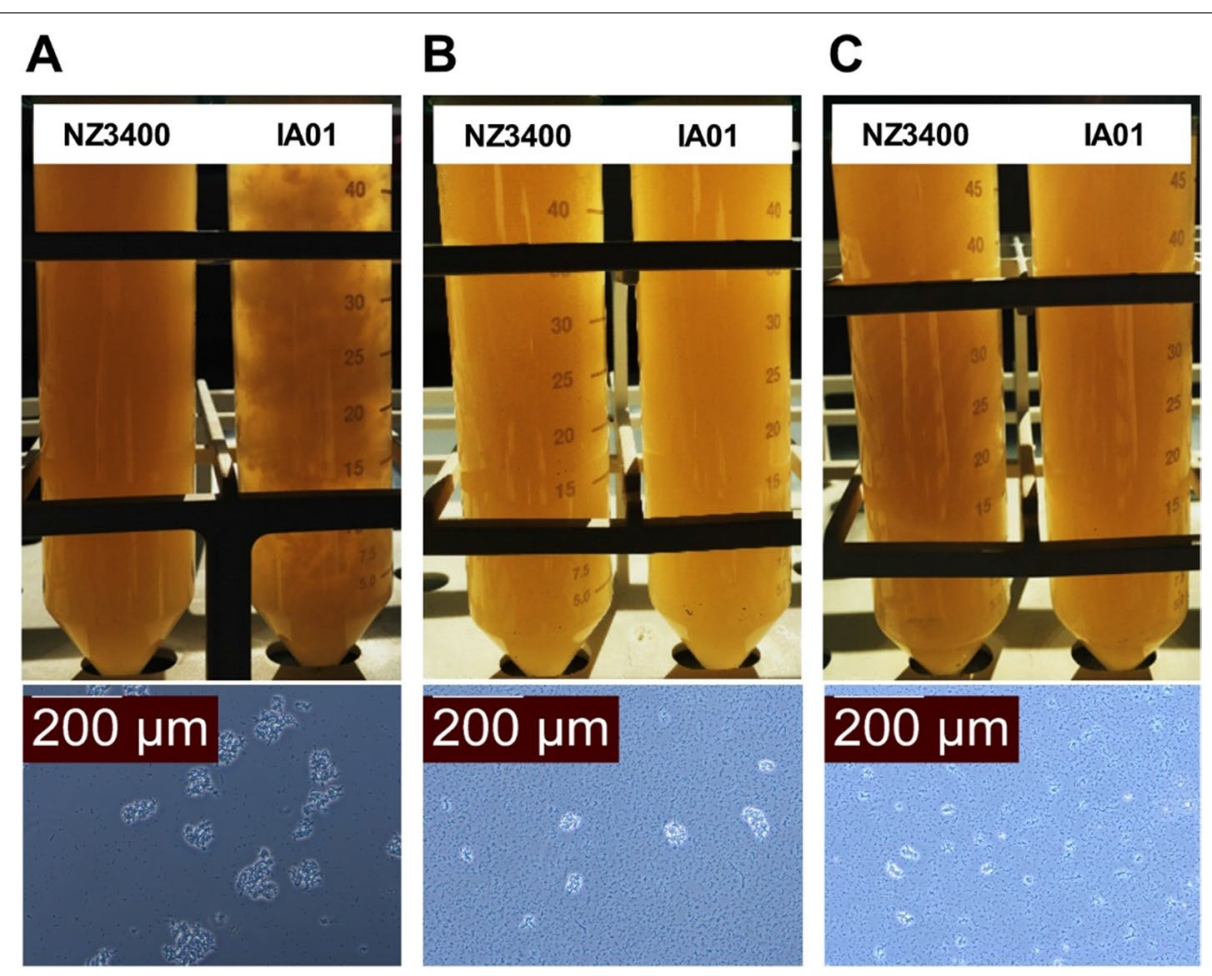

Fig. 3 Auto-aggregation phenotype diminishes during consecutive cultivation in MRS broth. Visual comparison between L. plantarum NZ3400 (left tube) and IA01 (right tube) and microscopic images of IA01 after the first (A), second (B) and third (C) consecutive culture in MRS broth. Results are representative for 15 tested L. plantarum cultures with auto-aggregation phenotype 
Table 1 L. plantarum NZ3400 derivative strains recovered from human in vitro microbiota

\begin{tabular}{|c|c|c|c|}
\hline Strain & Description & Auto-aggregation & Reference \\
\hline IA01 & NZ3400 derivative, $\mathrm{Cm}^{\mathrm{R}}$, C979T in LP_RS14990, isolated from in vitro fermentation & + & Isenring et al., 2021 [40] \\
\hline $\mid \mathrm{A} 01_{\mathrm{t} 0}$ & Strain IA01 grown after one overnight culture in MRS & + & This study \\
\hline $\mid \mathrm{A} 01_{\mathrm{t} 4}$ & Strain IA01 grown after four consecutive cultures in MRS & - & This study \\
\hline PA4_02 & NZ3400 derivative, $\mathrm{Cm}^{\mathrm{R}}$, isolated from in vitro fermentation & + & Isenring et al., 2021 [40] \\
\hline PA1.2_01 & $\begin{array}{l}\text { NZ3400 derivative, Cm }{ }^{R} \text {, C979T in LP_RS14990 and G382A in LP_RS01530, isolated from } \\
\text { in vitro fermentation }\end{array}$ & - & Isenring et al., 2021 [40] \\
\hline IA10 & NZ3400 derivative, $\mathrm{Cm}^{\mathrm{R}}, \mathrm{C} 569 \mathrm{~A}$ in LP_RS14255, isolated from in vitro fermentation & - & Isenring et al., 2021 [40] \\
\hline PA2_04 & NZ3400 derivative, $\mathrm{Cm}^{\mathrm{R}}$, C837A in LP_RS15205, isolated from in vitro fermentation & - & Isenring et al., 2021 [40] \\
\hline PA2_06 & NZ3400 derivative, $\mathrm{Cm}^{\mathrm{R}}$, C837A in LP_RS15205, isolated from in vitro fermentation & - & Isenring et al., 2021 [40] \\
\hline
\end{tabular}

192 significantly up- and 289 downregulated genes in IA01 compared to NZ3400 (FDR $<0.05$; Additional file 3: Table S1). In addition, IA01 was grown in four consecutive overnight cultures in MRS, which led to loss of the auto-aggregation phenotype, resulting in the strain IA $01_{\mathrm{t} 4}$. Comparison of aggregating $\mathrm{IAO}_{\mathrm{t} 0}$ and nonaggregating $\mathrm{IAO}_{\mathrm{t} 4}$ resulted in 583 significantly up- and 632 downregulated genes in IA01 ${ }_{\mathrm{t} 0}$ (Additional file 4: Table S2). Further, 94 up- and 123 downregulated genes were shared in both comparisons, being primary candidate genes for involvement in auto-aggregation. To narrow down these candidate genes, RNA sequencing data of the auto-aggregating strain PA4_02 and the wild type strain NZ3400 were compared. A total of 138 genes were significantly upregulated and 143 were significantly downregulated in PA4_02 (Additional file 5: Table S3). Combining all three comparisons revealed 50 significantly up- and 62 downregulated genes in auto-aggregating strains versus non-aggregating strains (Additional file 6: Table S4). Thereof, only genes with a $\mid \log _{2}$ ratio $\mid>1$ amongst all three comparisons were considered as possible candidate genes involved in autoaggregation (Table 2). Up-regulated genes in this data set encoded for two hypothetical proteins, three proteins with domains of unknown function (DUF), a haloacid dehalogenase-like hydrolase (HAD), the Multiple Antibiotic Resistance Regulator (MarR), and a mucin-binding protein (MucBP) domain (Table 2). The latter is known to be a mannose-specific adhesion protein encoded by msa [42]. The MarR family transcriptional regulator (LP_RS05230, marR) and the MucB domain-containing protein (LP_RS05225, msa) were strongest regulated by far with a log-fold change of 4.5-5.4 and 4.1-4.4, respectively (Table 2). The marR gene is located $11 \mathrm{bp}$ downstream of the $m s a$ gene and in the same direction, suggesting that they belong to one operon.

Table 2 Genes significantly up- and down-regulated ( $\mid$ Ratio $\left(\log _{2}\right) \mid>1$ ) in auto-aggregating compared to non-aggregation strains*

\begin{tabular}{|c|c|c|c|c|}
\hline \multirow[t]{2}{*}{ ORF } & \multirow[t]{2}{*}{ Product } & \multicolumn{3}{|c|}{ Ratio $\left(\log _{2}\right)$} \\
\hline & & $\overline{\text { PA4_02 }}$ & $\mid A 01_{t 0}^{b)}$ & $\mid A 01_{t 0}{ }^{c)}$ \\
\hline \multicolumn{5}{|l|}{ Upregulated } \\
\hline LP_RS05230 & MarR family transcriptional regulator & -5.17 & -4.54 & -5.37 \\
\hline LP_RS05225 & MucBP domain-containing protein & -4.23 & -4.10 & -4.42 \\
\hline LP_RS02885 & Hypothetical protein & -2.17 & -1.54 & -1.25 \\
\hline LP_RS10335 & DUF4355 domain-containing protein & -2.04 & -1.53 & -1.29 \\
\hline LP_RS02870 & DUF4355 domain-containing protein & -1.90 & -1.18 & -1.22 \\
\hline LP_RS10300 & Hypothetical protein & -1.55 & -1.03 & -1.07 \\
\hline LP_RS14830 & HAD family hydrolase & -1.52 & -1.11 & -1.28 \\
\hline LP_RS10310 & DUF3168 domain-containing protein & -1.49 & -1.58 & -1.20 \\
\hline \multicolumn{5}{|l|}{ Downregulated } \\
\hline LP_RS01470 & Hypothetical protein & 1.05 & 1.27 & 1.22 \\
\hline LP_RS00795 & Cna B-type domain-containing protein & 1.08 & 1.02 & 1.04 \\
\hline
\end{tabular}

${ }^{a}$ Auto-aggregating PA4_02 compared to the wild type NZ3400

${ }^{\mathrm{b}}$ Auto-aggregating IA01t $\mathrm{t}_{0}$ compared to $\mathrm{IAO}_{\mathrm{t}} \mathrm{t}_{4}$

CAuto-aggregating IA01 $\mathrm{t}_{0}$ compared to the wild type NZ3400

*phage-related genes were omitted. ORF: open reading frame 
Further, 2 genes encoding a hypothetical protein and Can B-type domain-containing protein, were significantly down-regulated in all three analyses (Table 2). However, the magnitude of change (maximum of 1.27 log-fold change) was small compared to the msa-marR operon (Table 2).

\section{DNA inversion predominates in auto-aggregating $L$. plantarum strains}

The very strong regulation of the msa-marR operon makes this operon the primary candidate responsible for the auto-aggregation phenotype. The msa promoter region contains two repeats that can invert, thereby changing the promoter activity of the operon [41]. To test whether such inversion occurred in autoaggregating strains, the RNAseq reads were plotted on the genome of WCFS1 and checked carefully for any genetic reorganization in the $m s a$ region. Indeed, an alternative junction was identified in all samples of the aggregating strains $I A 01_{t 0}$ and PA4_02, exactly at the repeat in the promoter of $m s a$. This new junction was not identified in the transcriptome samples from the wild type, IA $01_{t 4}$, nor in the samples of a previously constructed non-aggregating $\operatorname{lam} C$ knockout strain $L$. plantarum $\Delta$ LP_RS14990 [40] (Isenring, unpublished results).

Next, we quantified the occurrence of the inverse promoter using the RNAseq data reads. The ratio between the native and the inverted promoter was $8.3 \pm 1.2$ in the wild type and $9.5 \pm 1.4$ in the $\Delta \mathrm{LP}_{-}$ RS14990 strain, showing high prevalence of the native promoter in both non-aggregating strains. In contrast, the auto-aggregating strains $\mathrm{A} 01_{\mathrm{t} 0}$ and PA4_02 had a significantly lower ratio of $0.56 \pm 0.04$ and $0.54 \pm 0.07$ than the wild type strain and $\Delta$ LP_RS14990 $(p=0.001)$. Strain $\mathrm{IA}_{01} 1_{\mathrm{t} 4}$ lost its auto-aggregating phenotype and had a significantly lower a ratio of $5.3 \pm 0.9$ than the wild type $(p=0.03)$ suggesting that the inverted promoter is still present in strains that have lost the phenotype recently.

Last, we investigated whether the inverted promoter region is already present in a subpopulation of the wild type strain and in the non-aggregating strain IA10 (Table 1). A PCR specific for the native promoter resulted in amplification for the wild type strain NZ3400, strain IA10 and for both aggregating strains PA4_02 and AI01 (Additional file 7: Figure S3), supporting that the native promoter is present in all $L$. plantarum cultures. However, the PCR specific for the inverted promoter did not result in amplification for the wild type and IA10 strains but produced a clear amplicon in the cultures of PA4_02 and IA01.

\section{Discussion}

Auto-aggregation is a desired property for probiotics which is associated with enhanced colonization, inhibition of pathogenic infections and immunomodulation of the intestinal mucosa $[12,13,28,43,44]$. In this study, we describe for the first time the emerge of an auto-aggregating phenotype in the non-aggregating $L$. plantarum NZ3400 after exposure to modelled human colonic microbiota. Percentage of auto-aggregation was highest for $L$. plantarum isolated from reactor biofilms. Biofilm formation can be facilitated by autoaggregation [5, 45], however, quantification of biofilm formation on a polystyrene surface via crystal violet showed that biofilm formation of the aggregating strain PA4-02 was only enhanced compared to the wild type in minimal medium containing maltose. Aggregates were reported to form a multi-species cell-network that facilitates nutrient exchange, provides protection and creates a novel niche that aids microbiota colonization [23, 46]. L. plantarum cells in aggregates might therefore be able to survive better during starvation. It should be noted that starvation did not induce changes in propionate and butyrate concentrations and glucose, galactose and lactate were not detectable in both, control, and starved conditions. Therefore, these factors and the $\mathrm{pH}$, which was kept constant during fermentation, are not explaining the effects observed on aggregation.

Transcriptome analysis of the wild type strain and auto-aggregating $L$. plantarum strains showed that the genes encoded by LP_RS05225 ( $m s a$ ) and LP_RS05230 (marR) were both highly overexpressed in the autoaggregation phenotype. LP_RS05230 (previously known as lp_1230) is a transcriptional regulator that is located 11 base pairs downstream of LP_RS05225. In silico analysis of the genes showed that they form a bicistronic operon [41, 42], which is also supported by our data. The $m s a$ gene was identified as mannose-specific adhesin gene and is responsible for the agglutination of $L$. plantarum with Saccharamyces cerevisiae [41, 42]. Mannosespecific adherence mediates adherence of $L$. plantarum to human colonic cell line HT-29, epithelial Caco-2 cells and intestinal mucus in rats and thus assists in intestinal colonization [41, 47, 48]. In addition, pathogens such as Vibrio cholerae, Salmonella or Pseudomonas aeruginosa bind to mannose-containing glycoconjugates on the host intestinal surface [49-51]. Adherence of L. plantarum by mannose-specific adhesins to such glycoconjugates might therefore prevent binding of the pathogens. Furthermore, mannose-specific adhesins reduce pathogen colonization due to co-aggregation with mannose-containing surface structures of the pathogen [52-54]. Hence, activation of $m s a$ may be a desirable property for probiotics. 
Identification of marR and msa did not reveal the mechanism sustaining the auto-aggregation phenotype, since genome sequencing did not reveal any mutation or other variation in the auto-aggregating strains. However, plotting RNAseq reads on the wild type genome revealed two novel DNA-junctions in the promoter region of LP RS05225, that apparently could only be detected with the high read numbers produced in RNAseq experiments and the dedicated breseq software with a low detection limit for identification of variations in the genome [55]. The junction is identical to a previously reported inversion that is responsible for a strong yeast-agglutination phenotype in L. plantarum WCFS1, the parental strain of NZ3400 [41]. The inversion causes prevention of a stemloop structure increasing both, transcription and translation of the msa gene. The inversion was predominant in aggregation cultures and still more present in IA01 $1_{\mathrm{t} 4}$ than in non-aggregating stains. The occurrence of the aggregating phenotype over several generations can be explained by the presence of this relatively stable inversion. Moreover, the detected remains of the inversion in cultures that lost their aggregation phenotype may explain the rapid reverse of these cultures to an aggregating phenotype under selected conditions.

Aggregation was activated in absence of detectable glucose in fermentation reactors and in minimal medium without glucose. In addition, aggregation disappeared in glucose containing MRS medium. This suggests a role of glucose mediated catabolite repression via the canonical regulatory carbon catabolite protein A (CcpA) [56]. CcpA binds to catabolite response elements (cre) in the proximity to the regulated promoter, thereby regulating gene expression [57]. However, involvement of CcpA seems unlikely because reestablishment of auto-aggregation also occurred in the glucose-containing EMS medium. Further, no cre-elements were identified in the promoter region of $m s a$ and transcriptome analysis of a L. plantarum WCFS1 ccpa-knockout strain did not reveal differential expression in LP_RS05225 and LP_ RS05230 [58]. Other regulators might be involved in the msa promoter inversion, yet the exact regulation remains unclear.

The observed loss and reestablishment of the autoaggregation phenotype could be explained by phase variation, which leads to an heterogenous culture via an $\mathrm{ON} /$ OFF switch [59]. DNA inversions cause phase-variable surface protein expression [60-66] partially via genetic modifications by the activity of recombinases $[60,61$, 67-70]. Indeed, recombinases lead to DNA inversion in Bacteroides fragilis resulting in phase-variable expression of surface proteins responsible for auto-aggregation [71]. The higher expression of the recombinase RecT (Additional file 6: Table S4) in auto-aggregating strains might induce DNA inversion in the upstream region of $m s a$. However, classical phase variation in the sense of an $\mathrm{ON}$ OFF switch does not explain the slow disappearance of auto-aggregation during three consecutive MRS cultures.

Alternatively, growth in MRS medium might select for the native promoter, which is shown by the presence of the native orientation in the aggregating strains IA01 and PA4_02 (Additional file 7: Figure S3). Overexpression of the mannose-specific adhesin is toxic in L. plantarum $299 \mathrm{v}$, but not WCFS1, yet might lead to slower growth [41], explaining the vanishing of the auto-aggregating phenotype. Growth in MRS medium can then select for the fast-growing, non-aggregating fraction resulting in outcompeting the aggregating fraction during consecutive culturing. Slower growth of L. plantarum on sucrose, maltose [58] and EMS (data not shown) would allow outgrowth of the auto-aggregating fraction. Strong supporting evidence for this explanation is the difference in inverse and native promoter prevalence between the wild type and auto-aggregating variants.

The presence of both promoter orientations in aggregating strain cultures shows that an aggregating subculture proliferates in the fermenter. The inverse promoter orientation could not be detected in non-aggregating strains, suggesting strongly that the inversion is not present in non-aggregating cultures but is rather a relatively rare event and must be selected for to become visible. However, whether the inversion occurs spontaneously or is induced by the fermenter environment remains unclear.

\section{Conclusion}

We showed that cultivation of L. plantarum in a modelled in vitro human colonic microbiota leads to an autoaggregating subpopulation. This novel auto-aggregating phenotype is caused by a high expression of the mannose-specific adhesion gene msa. Both, auto-aggregation and mannose-mediated adhesion are desired properties of probiotics. Our data demonstrate that the PolyFermS fermentation model is suitable to detect and select for novel phenotypes related to the intestinal environment. This could further be applied to elucidate phenotypic adaptations and their underlying mechanisms of both probiotics and gut pathogens.

\section{Methods}

\section{Strains and growth conditions}

L. plantarum NZ3400, a WCFS1 derivative harboring a chloramphenicol resistance cassette in a neutral locus on the chromosome, was used as wild type strain [72]. NZ3400 derivative strains were recovered from in vitro human colonic microbiota after cultivation for up to 100 generations as described previously [40] (Table 1). 
L. plantarum strains were cultivated in De Man, Rogosa and Sharpe (MRS, Labo-Life Sàrl, Pully, Switzerland) broth at $37^{\circ} \mathrm{C}$, overnight, unless stated otherwise. To investigate the influence of different carbon sources on auto-aggregation, a minimal medium was designed based on the MRS composition [73] by omitting beef extract, peptone, glucose, Tween 80 and reducing yeast extract from 0.4 to $0.2 \%(\mathrm{w} / \mathrm{v})$. This minimal medium did not support L. plantarum growth and metabolic activity. Glucose, sucrose, maltose and fructose were added as individual carbon source at $1 \%(\mathrm{w} / \mathrm{v})$. In addition, the previously designed Effluent-MacFarlane-Sugar (EMS) medium [40], mimicking the abiotic environment of in vitro colonic microbiota, was used to test the effect on auto-aggregation in absence of microbiota. EMS medium consisted of sterile filtered effluent from the in vitro colonic microbiota, MacFarlane medium [74], which mimics the chyme entering the colon (ratio 9:1), and glucose $(0.75 \%, \mathrm{w} / \mathrm{v})$.

\section{Establishment of a high-throughput screening method for auto-aggregation}

A high-throughput screening method to detect autoaggregating $L$. plantarum strains from colonic in vitro human gut microbiota was developed by modifying an existing agglutination assay [41]. MRS broth in 96-well tissue culture test plates (Bioswisstec AG, Schaffhausen, Switzerland) was inoculated with L. plantarum strains and grown overnight. Cultures were analyzed microscopically in biological triplicates (Leica DM1000, Leica Microsystems, Wetzlar, Germany) for aggregate formation.

\section{Characterization of $L$. plantarum auto-aggregation in the in vitro colonic microbiota}

The design and implementation of the continuous intestinal fermentation model PolyFermS was presented in detail previously [40]. In short, the inoculum reactors (IR) of four models were inoculated $(30 \%, \mathrm{v} / \mathrm{v})$ with distinct fecal microbiota from healthy adults immobilized in polysaccharide gel beads. A detailed description of the PolyFermS set-up and conditions, microbiota compositions and fermentation profiles are reported in Isenring et al. [40]. The four IRs used for auto-aggregation experiments were operated for time periods ranging from 10 to 72 days. Second-stage treatment reactors (TRs) were continuously inoculated by IR effluent (5\%) and fed with fresh MacFarlane medium (95\%), formulated to mimic the chime entering the colon [74]. Metabolite concentrations of the continuous fermentation were determined by high-performance liquid chromatography as described previously [40]. To investigate the influence of colonic microbiota on auto-aggregation, L. plantarum NZ3400 was supplemented to TRs connected to the IR and operated with identical conditions. Derivatives were isolated from the effluent after 10 days from three TRs containing microbiota of donor 1, five TRs of donor 2, five TRs of donor 3 and six of donor 4 .

L. plantarum strains were recovered from the biofilm in three reactors collected at the end of the fermentation containing microbiota of donor 2 as described previously [40] and compared to L. plantarum isolated from reactor effluent harvested on the same day. Nutritive stress was simulated by starvation of the modelled gut microbiota by medium inflow interruption. L. plantarum strains were recovered before and $24 \mathrm{~h}$ after starvation.

\section{Biofilm assay}

Biofilm formation capability of the non-aggregating strain NZ3400 and its aggregating derivative strain PA4_02 was assessed in MRS, minimal medium containing glucose, sucrose, fructose or maltose as sole carbon source and EMS medium as described previously with minor modifications [75]. Wells of 96-well tissue culture test plates (Bioswisstec AG, Schaffhausen, Switzerland) containing $200 \mu \mathrm{l}$ of corresponding growth medium were inoculated with $10^{6} \mathrm{CFU} / \mathrm{ml} L$. plantarum. Wells only containing the medium served as control. The plates were incubated at $37^{\circ} \mathrm{C}$ for $24 \mathrm{~h}$. The liquid was removed, and the wells were washed three times with phosphate-buffered saline (PBS), pH6.2. After drying, the wells were supplemented with crystal violet $(0.1 \%, \mathrm{w} / \mathrm{v})$ and incubated for $30 \mathrm{~min}$. The stained biofilm was washed three times with PBS, the dye was resolved in ethanol (99\%) and absorbance was measured at $595 \mathrm{~nm}$ (PowerWaveTMXS; Bio-Tek Instrument Inc., Winooski, VT, USA).

\section{Stability of auto-aggregation phenotype}

The stability of auto-aggregation in $15 \mathrm{~L}$. plantarum isolates recovered from all four microbiota was tested in three consecutive overnight cultures in MRS broth inoculated at $1 \%(\mathrm{v} / \mathrm{v})$, corresponding to approximately 25 generations. Auto-aggregation was assessed visually and microscopically.

\section{RNA isolation and sequencing}

RNA sequencing was performed on the wild type strain L. plantarum NZ3400, the auto-aggregating strains IA0 ${ }_{\mathrm{t} 0}$ and PA4_02 and the non-aggregating IA0 ${ }_{\mathrm{t} 4}$. Precultures were done in MRS broth at $30^{\circ} \mathrm{C}$ overnight. Experiments were performed in triplicates for NZ3400, IA $01_{\mathrm{t} 0}$ and $\mathrm{IAO} 1_{\mathrm{t} 4}$ and in duplicates for PA4_02. Cultures were inoculated from the pre-culture and grown until $\mathrm{OD}_{600 \mathrm{~nm}}=2.6-2.7$, with final measurement of the $\mathrm{pH}$, glucose utilization and lactate production. Total RNA was extracted based on chloroform/phenol extraction 
followed by purification using the High Pure RNA isolation kit (Roche Diagnostics, Rotkreuz, Switzerland) as described previously (58). RNA quantity, purity and integrity were verified using an Agilent 2200 TapeStation (Agilent Technologies, Santa Clara, CA, USA). Samples with an RNA integrity number (RIN) $>9$ and a $16 \mathrm{~S} / 23 \mathrm{~S}$ rRNA ratio $>1.5$ were selected for rRNA depletion. Thereafter, EDTA was added to $1 \mathrm{mM}$ and depletion was performed using the MICROBExpress ${ }^{\mathrm{TM}}$ Bacterial mRNA Enrichment Kit (Life Technologies Europe BV, Zug, Switzerland) following the manufacturer's instructions. Concentrations of depleted samples were determined in a TapeStation and normalized to $100 \mathrm{ng} / \mu \mathrm{l}$ using in Tris$\mathrm{HCl}(10 \mathrm{mM}, \mathrm{pH}=8.5)$.

Sequencing of $100 \mathrm{bp}$ single reads was done on Illumina Novaseq 6000 (Illumina Inc., California, USA) at the Functional Genomics Center Zurich (FGCZ). The library was prepared according to the Illumina Truseq Total RNA protocol.

\section{Analyses of DNA inversion upstream of LP_RS05225}

The pipeline for the analysis of short-read re-sequencing breseq [76] was used to identify possible new junctions in the upstream region of $m s a$ (LP_RS05225). A 9439-bp fragment of the WCFS1 genome $7000 \mathrm{bp}$ upstream and 2000 bp downstream of the marR gene (LP_RS05230) was used as reference and the RNAseq reads as re-sequencing reads. Standards settings were applied, and each set of reads was analyzed separately.

To quantify the amount of standard and reversed promoter regions of $m s a$ in the strains, a fragment containing the 234-bp upstream regions of the msa start with both promotor orientations was constructed and stored as two sequences in a single fasta file. RNAseq reads were plotted to the sequences using Bowtie2 [77] with standard settings. The Bowtie2 output was further processed to a reads-per-gene spreadsheet as described above. The ratio between both promoter regions was calculated and averaged. A two-tailed t-test was used to calculate the $p$ value.

To identify a possible subpopulation harboring the msa-MarR-inversion, primers were designed (Additional file 8: Figure S4) to amplify the native sequence (Native_fw: $\quad 5^{\prime}$-GGGAGTAAAGCGTGCAATGT-3'; Native_rev: 5'-GCATTACCTATTTGATAACGCAGA $-3^{\prime}$ ) and the inverted sequence (Inversion_fw: $5^{\prime}$-TCA TGCGAAAGGATAGGTGTAA-3'; Inversion_rev:5'TTGAGATGCTGAATCGTTCG-3') in the promoter region. DNA of the non-aggregating wild type NZ3400 and IA10, and the aggregating PA4_02 and IA01 was extracted as described previously using lysozyme-celllysis and purification using the Wizard Genomic DNA purification kit (Promega, Dübendorf, Switzerland) [40].
PCR reactions were performed in a volume of $25 \mu \mathrm{l}$ containing $20 \mathrm{ng}$ DNA, $12.5 \mu \mathrm{l} 2 \mathrm{x}$ PCR Master Mix (Fermentas, Le Mont-sur-Lausanne, Switzerland), $1 \mu \mathrm{M}$ of each primer (Microsynth, Balgach, Switzerland) and sterile, DNase-free water (Fermentas). PCR was performed with an initial denaturation $\left(95^{\circ} \mathrm{C}, 2 \mathrm{~min}\right)$, followed by 30 or 40 cycles of denaturation $\left(95^{\circ} \mathrm{C}, 30 \mathrm{~s}\right)$, annealing $\left(51^{\circ} \mathrm{C}\right.$, $30 \mathrm{~s})$ and replication $\left(72^{\circ} \mathrm{C}, 25 \mathrm{~s}\right)$ and subsequent final replication $\left(72{ }^{\circ} \mathrm{C}, 7 \mathrm{~min}\right)$ in a Biometra ${ }^{\circledR} \mathrm{T} 3000$ Thermocycler (Labgene, Châtel-Saint-Denis, Switzerland). Amplified products and DNA marker (100 bp, BioConcept, Allschwil, Switzerland) were analyzed via agarose $(2 \%, \mathrm{w} / \mathrm{v})$ gel electrophoresis and visualized with gel red.

\section{Data analysis}

Graphs were created using GraphPad Prism ${ }^{\circledR}$ version 8 (GraphPad Software Inc., San Diego, CA, USA). Analysis of RNAseq reads was performed as described previously [78]. Shortly, reads were mapped on the chromosome and plasmid of L. plantarum WCFS1 (accession numbers NC_006375.1, NC_006376.1, NC_006377.1, and NC_004567.2) using Bowtie2. Data filtering, normalization and analysis was done in $\mathrm{R}$ (version 3.6.2) using the packages DESeq and EdgeR. Only genes with a false discovery rate $(\mathrm{FDR})<0.05$ and a differential expression of minimum 2 fold ( $\mid$ ratio $\left(\log _{2}\right)>1 \mid$ ) were considered as significant differently expressed.

\section{Supplementary Information}

The online version contains supplementary material available at https://doi. org/10.1186/s12866-021-02331-x.

Additional file 1: Figure S1. Biofilm formation ability of the nonaggregating wild type strain L. plantarum NZ3400 (blue) and the isogenic aggregating strain PA4_02 (red). Biofilm formation was assessed in MRS and EMS medium and minimal medium containing sucrose ( $\mathrm{mm}$-sucrose) fructose (mm-fructose), glucose (mm-glucose) or maltose (mm-maltose) as carbon source. Data represent mean value \pm standard deviation of biological triplicates.

Additional file 2: Figure S2. Fermentation metabolite profile before (blue) and after (red) microbiota starvation. Bars represent mean \pm standard deviation of metabolite abundance relative to the total produced metabolites of six parallel operated TRs the day before and $24 \mathrm{~h}$ after starvation of the gut microbiota. Significance was calculated by pairedsample $t$ test: ${ }^{* *}<0.01,{ }^{* * * *} p<0.0001$.

Additional file 3: Table S1. Significantly up- and downregulated genes in the auto-aggregating L. plantarum IA01 compared to NZ3400.

Additional file 4: Table S2. Significantly up- and downregulated genes in the auto-aggregating L. plantarum IA01 $\mathrm{t}_{0}$ compared to the non-aggregating $\mid \mathrm{AO}_{0} \mathrm{t}_{4}$.

Additional file 5: Table S3. Significantly up- and downregulated genes in the auto-aggregating L. plantarum PA4_02 compared to NZ3400.

Additional file 6: Table S4. Significantly up- and downregulated genes in all auto-aggregating L. plantarum.

Additional file 7: Figure S3. Visualization of DNA fragments (agarose gel, 2\%) obtained by PCR amplification using 30 (A) and 40 (B) cycles of 
denaturation, annealing and replication. NZ3400: L. plantarum wild type strain (non-aggregating); IA10: L. plantarum recovered from the gut microbiota (non-aggregating); PA4_02 and IA01: L. plantarum recovered from the gut microbiota (auto-aggregating).

Additional file 8: Figure S4. Scheme of the PCR-set-up to detect the native and inversed msa-marR promoter region. A) Native orientation: DNA will be amplified using the primer combination Native_fw/ Native_rev, resulting in a $225 \mathrm{bp}$ amplicon. B) Inverted DNA will be amplified using the primer combination Inverse_fw/Inverse_rev, resulting in a 313 bp amplicon. P: promoter.

\section{Acknowledgements}

We thank Alfonso Die for assistance during HPLC measurements and LuC Jaquenod and Ilsabe Wiebecke for experimental assistance.

\section{Authors' contributions}

J.I., A.G., C.L. and M.J.A.S. designed the study. Experiments were performed by J.I. Data analyses were done by J.I. and M.J.A.S. J.I. and M.J.A.S. drafted the manuscript and all authors critically reviewed the manuscript. The authors read and approved the final manuscript.

\section{Funding}

This study was funded by the ETH Zurich research grant program (ETH-42 16-1).

\section{Availability of data and materials}

Gene expression data are directly accessible through GEO (series accession number GSE172351, https://www.ncbi.nlm.nih.gov/geo/query/acc.cgi?acc= GSE172351).

\section{Declarations}

\section{Ethics approval and consent to participate}

This study was exempted from review by the Ethics Committee of ETH Zurich because sample collection procedure has not been performed under intervention conditions. Informed written consent was obtained from fecal donors.

\section{Consent for publication}

Not applicable.

\section{Competing interests}

Not applicable.

\section{Author details}

'Laboratory of Food Biotechnology, Institute of Food, Nutrition and Health, ETH Zurich, Schmelzbergstrasse 7, 8092 Zürich, Switzerland. ${ }^{2}$ Institute for Food Hygiene and Safety, University of Zürich, Zurich, Switzerland.

Received: 3 June 2021 Accepted: 24 September 2021 Published online: 05 October 2021

\section{References}

1. Puebla-Barragan S, Reid G. Forty-five-year evolution of probiotic therapy. Microb Cell. 2019;6:184-96.

2. Hill C, Guarner F, Reid G, Gibson GR, Merenstein DJ, Pot B, et al. Expert consensus document. The International Scientific Association for Probiotics and Prebiotics consensus statement on the scope and appropriate use of the term probiotic. Nat Rev Gastroenterol Hepatol. 2014;11:506-14.

3. Tuomola E, Crittenden R, Playne M, Isolauri E, Salminen S. Quality assurance criteria for probiotic bacteria. Am J Clin Nutr. 2001;73:393-8.

4. Charteris WP, Kelly PM, Morelli L, Collins JK. Development and application of an in vitro methodology to determine the transit tolerance of potentially probiotic Lactobacillus and Bifidobacterium species in the upper human gastrointestinal tract. J Appl Microbiol. 1998;84:759-68.
5. Rickard AH, Gilbert P, High NJ, Kolenbrander PE, Handley PS. Bacterial coaggregation: an integral process in the development of multi-species biofilms. Trends Microbiol. 2003;11:94-100.

6. Caldwell DE, Atuku E, Wilkie DC, Wivcharuk KP, Karthikeyan S, Korber DR, et al. Germ theory vs. community theory in understanding and controlling the proliferation of biofilms. Adv Dent Res. 1997;11:4-13.

7. Eboigbodin KE, Newton JRA, Routh AF, Biggs CA. Role of nonadsorbing polymers in bacterial aggregation. Langmuir. 2005;21:12315-9.

8. Cesena C, Morelli L, Alander M, Siljander T, Tuomola E, Salminen S, et al. Lactobacillus crispatus and its nonaggregating mutant in human colonization trials. Int J Dairy Sci. 2001;84:1001-10.

9. Reniero R, Cocconcelli P, Bottazzi V, Morelli L. High-frequency of conjugation in Lactobacillus mediated by an aggregation-promoting factor. J Gen Microbiol. 1992;138:763-8.

10. Janković T, Frece J, Abram M, Gobin I. Aggregation ability of potential probiotic Lactobacillus plantarum strains. Int J Sanit Eng Res. 2012;6:19-24.

11. Gomaa EZ. Antimicrobial and anti-adhesive properties of biosurfactant produced by lactobacilli isolates, biofilm formation and aggregation ability. J Gen Appl Microbiol. 2013;59:425-36.

12. Garcia-Cayuela T, Korany AM, Bustos I, de Cadinanos LPG, RequenaT, Pelaez C, et al. Adhesion abilities of dairy Lactobacillus plantarum strains showing an aggregation phenotype. Food Res Int. 2014;57:44-50.

13. Collado MC, Meriluoto J, Salminen S. Adhesion and aggregation properties of probiotic and pathogen strains. Eur Food Res Technol. 2008;226:1065-73.

14. Del Re B, Sgorbati B, Miglioli M, Palenzona D. Adhesion, autoaggregation and hydrophobicity of 13 strains of Bifidobacterium longum. Lett Appl Microbiol. 2000;31:438-42.

15. Tulumoglu S, Yuksekdag ZN, Beyatli Y, Simsek O, Cinar B, Yasar E. Probiotic properties of lactobacilli species isolated from children's feces. Anaerobe. 2013;24:36-42.

16. Vlkova E, Rada V, Smehilova M, Killer J. Auto-aggregation and co-aggregation ability in bifidobacteria and clostridia. Folia Microbiol. 2008;53:263-9.

17. Topcu KC, Kaya M, Kaban G. Probiotic properties of lactic acid bacteria strains isolated from pastirma. LWT. 2020;134:110216.

18. Fonseca HC, Melo DD, Ramos CL, Dias DR, Schwan RF. Probiotic properties of lactobacilli and their ability to inhibit the sdhesion of enteropathogenic bacteria to Caco-2 and HT-29 cells. Probiotics Antimicro. 2021;13:102-12.

19. Wang J, Wang J, Yang K, Liu MM, Zhang J, Wei XY, et al. Screening for potential probiotic from spontaneously fermented non-dairy foods based on in vitro probiotic and safety properties. Ann Microbiol. 2018;68:803-13.

20. Binti Wan Mohd Noor WSA. An investigation of the phenotypic and genotypic changes in Pseudomonas putida CP1 following substratedependent autoaggregation. Dublin: Dublin City University; 2013.

21. Zuo FL, Yu R, Xiao M, Khaskheli GB, Sun XF, Ma HQ, et al. Transcriptomic analysis of Bifidobacterium longum subsp longum BBMN68 in response to oxidative shock. Sci Rep. 2018;8:1-12.

22. Hevia A, Martinez N, Ladero V, Alvarez MA, Margolles A, Sanchez B. An extracellular serine/threonine-rich protein from Lactobacillus plantarum NCIMB 8826 is a novel aggregation-promoting factor with affinity to mucin. Appl Environ Microbiol. 2013;79:6059-66.

23. TrunkT, Khalil HS, Leo JC. Bacterial autoaggregation. AIMS Microbiol. 2018;4:140-64.

24. Bossier P, Verstraete W. Triggers for microbial aggregation in activated sludge? Appl Microbiol Biot. 1996;45:1-6.

25. Farrell A, Quilty B. Substrate-dependent autoaggregation of pseudomonas putida CP1 during the degradation of mono-chlorophenols and phenol. J Ind Microbiol Biot. 2002;28:316-24.

26. Perez PF, Minnaard Y, Disalvo EA, De Antoni GL. Surface properties of bifidobacterial strains of human origin. Appl Environ Microb. 1998;64:21-6.

27. Wadstrom T, Andersson K, Sydow M, Axelsson L, Lindgren S, Gullmar B. Surface properties of lactobacilli isolated from the small intestine of pigs. J Appl Bacteriol. 1987;62:513-20.

28. Goh YJ, Klaenhammer TR. Functional roles of aggregation-promotinglike factor in stress tolerance and adherence of Lactobacillus acidophilus NCFM. Appl Environ Microb. 2010;76:5005-12. 
29. Boris S, Suarez JE, Barbes C. Characterization of the aggregation promoting factor from Lactobacillus gasseri, a vaginal isolate. J Appl Microbiol. 1997:83:413-20.

30. Malik S, Petrova MI, Claes IJJ, Verhoeven TLA, Busschaert P, Vaneechoutte $M$, et al. The highly autoaggregative and adhesive phenotype of the vaginal Lactobacillus plantarum strain CMPG5300 is sortase dependent. Appl Environ Microb. 2013;79:4576-85.

31. Krausova G, Hyrslova I, Hynstova I. In vitro evaluation of adhesion capacity, hydrophobicity, and auto-aggregation of newly isolated potential probiotic strains. Fermentation. 2019;5:100.

32. Spencer RJ, Chesson A. The effect of Lactobacillus spp. on the attachment of enterotoxigenic Escherichia coli to isolated porcine enterocytes. J Appl Bacteriol. 1994;77:215-20.

33. Nath S, Sikidar J, Roy M, Deb B. In vitro screening of probiotic properties of Lactobacillus plantarum isolated from fermented milk product. Food Qual Saf. 2020;4:213-23.

34. Abushelaibi A, Al-Mahadin S, El-Tarabily K, Shah NP, Ayyash M. Characterization of potential probiotic lactic acid bacteria isolated from camel milk. Lwt-Food Sci Technol. 2017;79:316-25.

35. Aziz G, Fakhar H, Rahman SU, Tariq M, Zaidi A. An assessment of the aggregation and probiotic characteristics of Lactobacillus species isolated from native (desi) chicken gut. J Appl Poult Res. 2019;28:846-57.

36. Sui Y, Liu J, Liu Y, Wang Y, Xiao Y, Gao B, et al. In vitro probiotic characterization of Lactobacillus strains from fermented tangerine vinegar and their cholesterol degradation activity. Food Biosci. 2021;39:100843.

37. Sheth $R U$, Li MQ, Jiang WQ, Sims PA, Leong KW, Wang HH. Spatial metagenomic characterization of microbial biogeography in the gut. Nat Biotechnol. 2019;37:877.

38. van den Nieuwboer M, van Hemert S, Claassen E, de Vos WM. Lactobacillus plantarum WCFS1 and its host interaction: a dozen years after the genome. Microb Biotechnol. 2016;9:452-65.

39. Kleerebezem M, Boekhorst J, van Kranenburg R, Molenaar D, Kuipers OP, Leer $\mathrm{R}$, et al. Complete genome sequence of Lactobacillus plantarum WCFS1. PNAS. 2003;100:1990-5.

40. Isenring J, Geirnaert A, Hall AR, Jans C, Lacroix C, Stevens MJA. In vitro gut modeling as a tool for adaptive evolutionary engineering of Lactiplantibacillus plantarum. mSystems. 2021;6:e01085-20.

41. Holst B, Glenting J, Holmstrom K, Israelsen H, Vrang A, Antonsson M, et al. Molecular switch controlling expression of the mannose-specific adhesin, Msa, in Lactobacillus plantarum. AEM. 2019;85:e02954-18.

42. Pretzer G, Snel J, Molenaar D, Wiersma A, Bron PA, Lambert J, et al. Biodiversity-based identification and functional characterization of the mannose-specific adhesin of Lactobacillus plantarum. J Bacteriol. 2005;187:6128-36.

43. Galdiero F, Romano Carratelli C, Nuzzo I, Bentivoglio C, Galdiero M. Phagocytosis of bacterial aggregates by granulocytes. Eur J Epidemiol. 1988:4:456-60.

44. Voltan S, Castagliuolo I, Elli M, Longo S, Brun P, D'Inca R, et al. Aggregating phenotype in Lactobacillus crispatus determines intestinal colonization and TLR2 and TLR4 modulation in murine colonic mucosa. Clin Vaccine Immunol. 2007;14:1138-48.

45. Sorroche FG, Spesia MB, Zorreguieta A, Giordano W. A positive correlation between bacterial autoaggregation and biofilm formation in native Sinorhizobium meliloti isolates from Argentina. Appl Environ Microb. 2012;78:4092-101.

46. Tytgat HLP, Nobrega FL, van der Oost J, de Vos WM. Bowel biofilms: tipping points between a healthy and compromised gut? Trends Microbiol. 2019:27:17-25.

47. Adlerberth I, Ahrne S, Johansson ML, Molin G, Hanson LA, Wold AE. A mannose-specific adherence mechanism in Lactobacillus plantarum conferring binding to the human colonic cell line HT-29. Appl Environ Microbiol. 1996:62:2244-51.

48. Sun J, Le GW, Shi YH, Su GW. Factors involved in binding of Lactobacillus plantarum Lp6 to rat small intestinal mucus. Lett Appl Microbiol. 2007:44:79-85.

49. Aslanzadeh J, Paulissen LJ. Role of type 1 and type 3 fimbriae on the adherence and pathogenesis of Salmonella enteritidis in mice. Microbiol Immunol. 1992;36:351-9.

50. Imberty A, Wimmerova M, Mitchell EP, Gilboa-Garber N. Structures of the lectins from Pseudomonas aeruginosa: insights into the molecular basis for host glycan recognition. Microbes Infect. 2004;6:221-8.
51. Bhattacharjee JW, Srivastava BS. Mannose-sensitive haemagglutinins in adherence of vibrio cholerae eltor to intestine. J Gen Microbiol. 1978:107:407-10.

52. Johansson ML, Nobaek S, Berggren A, Nyman M, Bjorck I, Ahrne S, et al. Survival of Lactobacillus plantarum DSM 9843 (299v), and effect on the short-chain fatty acid content of faeces after ingestion of a rose-hip drink with fermented oats. Int J Food Microbiol. 1998;42:29-38.

53. Wold AE, Thorssen M, Hull S, Eden CS. Attachment of Escherichia coli via mannose- or gal alpha1->4Gal beta-containing receptors to human colonic epithelial cells. Infect Immun. 1988;56:2531-7.

54. Leon-Romero A, Dominguez-Manzano J, Garrido-Fernandez A, ArroyoLopez FN, Jimenez-Diaz R. Formation of in vitro mixed-species biofilms by Lactobacillus pentosus and yeasts isolated from spanish-style green table olive fermentations. Appl Environ Microbiol. 2016;82:689-95.

55. Deatherage DE, Traverse CC, Wolf LN, Barrick JE. Detecting rare structural variation in evolving microbial populations from new sequence junctions using breseq. Front Genet. 2015;5:468.

56. Henkin TM, Grundy FJ, Nicholson WL, Chambliss GH. Catabolite repression of alpha amylase gene expression in Bacillus subtilis involves a transacting gene product homologous to the Escherichia coli lacl and galR repressors. Mol Microbiol. 1991;5:575-84.

57. Weickert MJ, Chambliss GH. Site-directed mutagenesis of a catabolite repression operator sequence in Bacillus subtilis. P Natl Acad Sci USA. 1990;87:6238-42

58. Stevens MJ. Transcriptome response of Lactobacillus plantarum to global regulator deficiency, stress and other environmental conditions. Netherlands: Wageningen University; 2008.

59. van der Woude MW. Epigenetic phase variation in bacterial pathogens. In: Doerfler W, Casadesús J, editors. Epigenetics of infectious diseases. Cham: Springer; 2017. p. 159-73.

60. Dybvig K. DNA rearrangements and phenotypic switching in prokaryotes Mol Microbiol. 1993;10:465-71.

61. Vanputte P, Goosen N. DNA inversions in phages and bacteria. Trends Genet. 1992;8:457-62.

62. Henderson IR, Owen P, Nataro JP. Molecular switches - the ON and OFF of bacterial phase variation. Mol Microbiol. 1999;33:919-32.

63. Dworkin J, Blaser MJ. Molecular mechanisms of campylobacter fetus surface layer protein expression. Mol Microbiol. 1997;26:433-40.

64. Lenich AG, Glasgow AC. Amino-acid-sequence homology between Piv, an essential protein in site-specific DNA inversion in Moraxella lacunata and transposases of an unusual family of insertion elements. J Bacteriol. 1994:176:4160-4.

65. Moses EK, Good RT, Sinistaj M, Billington SJ, Langford CJ, Rood JI. A multiple site-specific DNA inversion model for the control of Omp1 phase and antigenic variation in Dichelobacter nodosus. Mol Microbiol. 1995;17:183-96.

66. Bhugra B, Voelker LL, Zou NX, Yu HL, Dybvig K. Mechanism of antigenic variation in mycoplasma pulmonis: interwoven, site-specific DNA inversions. Mol Microbiol. 1995;18:703-14.

67. Rojo F, Alonso JC. A novel site-specific recombinase encoded by the streptococcus pyogenes plasmid pSM19035. J Mol Biol. 1994;238:159-72.

68. Alonso JC, Weise F, Rojo F. The Bacillus subtilis histone-like protein Hbsu is required for DNA resolution and DNA inversion mediated by the beta recombinase of plasmid pSM19035. J Biol Chem. 1995;270:2938-45.

69. Canosa I, Lurz R, Rojo F, Alonso JC. Beta recombinase catalyzes inversion and resolution between two inversely oriented six sites on a supercoiled DNA substrate and only inversion on relaxed or linear substrates. J Biol Chem. 1998:273:13886-91.

70. Hallet B, Sherratt DJ. Transposition and site-specific recombination: adapting DNA cut-and-paste mechanisms to a variety of genetic rearrangements. FEMS Microbiol Rev. 1997;21:157-78.

71. Weinacht KG, Roche H, Krinos CM, Coyne MJ, Parkhill J, Comstock LE. Tyrosine site-specific recombinases mediate DNA inversions affecting the expression of outer surface proteins of Bacteroides fragilis. Mol Microbiol. 2004;53:1319-30

72. Remus DM, van Kranenburg R, van Swam II, Taverne N, Bongers RS, Wels $\mathrm{M}$, et al. Impact of 4 Lactobacillus plantarum capsular polysaccharide clusters on surface glycan composition and host cell signaling. Microb Cell Factories. 2012:11:1-10.

73. De Man J, Rogosa D, Sharpe ME. A medium for the cultivation of lactobacilli. J Appl Microbiol. 1960;23:130-5. 
74. Macfarlane GT, Macfarlane S, Gibson GR. Validation of a three-stage compound continuous culture system for investigating the effect of retention time on the ecology and metabolism of bacteria in the human colon. Microb Ecol. 1998;35:180-7.

75. O'Toole GA, Pratt LA, Watnick PI, Newman DK, Weaver VB, Kolter R. Genetic approaches to study of biofilms. Biofilms. 1999;310:91-109.

76. Barrick JE, Colburn G, Deatherage DE, Traverse CC, Strand MD, Borges $\mathrm{J}$, et al. Identifying structural variation in haploid microbial genomes from short-read resequencing data using breseq. BMC Genomics. 2014;15:1039.
77. Langmead B, Salzberg SL. Fast gapped-read alignment with bowtie 2. Nat Methods. 2012;9:357-9.

78. Horlbog JA, Stevens MJA, Stephan R, Guldimann C. Global transcriptional response of three highly acid-tolerant field strains of listeria monocytogenes to $\mathrm{HCl}$ stress. Microorganisms. 2019;7:455.

\section{Publisher's Note}

Springer Nature remains neutral with regard to jurisdictional claims in published maps and institutional affiliations.
Ready to submit your research? Choose BMC and benefit from:

- fast, convenient online submission

- thorough peer review by experienced researchers in your field

- rapid publication on acceptance

- support for research data, including large and complex data types

- gold Open Access which fosters wider collaboration and increased citations

- maximum visibility for your research: over $100 \mathrm{M}$ website views per year

At BMC, research is always in progress.

Learn more biomedcentral.com/submissions 